\title{
Pooled analysis of mid-regional pro-adrenomedullin values in COVID-19 patients with critical illness
}

\author{
Giuseppe Lippi $^{1}\left[\right.$ [ $\cdot$ Brandon Michael Henry ${ }^{2}$
}

Received: 2 March 2021 / Accepted: 29 April 2021 / Published online: 7 May 2021

c) Società Italiana di Medicina Interna (SIMI) 2021

Keywords Coronavirus $\cdot$ COVID-19 $\cdot$ Mid-regional pro-adrenomedullin $\cdot$ MR-proADM

Dear Editor,

Adrenomedullin (ADM), a potent vasodilatory peptide hormone produced by endothelial cells and several other mammalian tissues (heart, lung, kidney, bone, adrenals, etc.) exerts a vast array of angiogenic, anti-inflammatory, antioxidant and anti-apoptotic activities, thus playing a crucial role in inflammatory diseases and influencing the progression from sepsis to septic shock [1]. Recent evidence suggests that the measurement of the mid-regional pro-adrenomedullin (MR-proADM), a 48 amino acid mostly inert fragment split from the final ADM, which is secreted in 1:1 ratio and which has a significantly longer half-life than ADM, may offer considerable clinical prognostic value for predicting the risk of developing critical illness in patients with overt sepsis [1,2], as well as in those with severe localized infections, such as community-acquired pneumonias [3], including those of viral origin [4]. As interstitial pneumonia is the leading pathological manifestation of coronavirus disease 2019 (COVID-19), and given that patients with COVID19 are at extremely high risk of developing bacterial superinfections, especially those needing intensive care [5], we performed a critical literature review and meta-analysis to explore as to whether MR-proADM assessment may help predicting unfavorable disease progression in COVID-9 patients.

An electronic search was carried out in PubMed, Scopus, and Web of Science, using the keywords "adrenomedullin" OR "proadrenomedullin" OR "Mid-regional

Giuseppe Lippi

giuseppe.lippi@univr.it

1 Section of Clinical Biochemistry, University Hospital of Verona, Piazzale L.A. Scuro, 10, 37134 Verona, Italy

2 Cardiac Intensive Care Unit, The Heart Institute, Cincinnati Children's Hospital Medical Center, Cincinnati, OH, USA
proAdrenomedullin" OR "MR-proADM" AND "coronavirus disease 2019" OR "COVID-19" within all fields, and without language or date limits (i.e., up to April 20, 2021). The two authors reviewed title, abstract and full text of all documents identified with these search criteria, selecting studies which described MR-proADM values in COVID19 patients with different degrees of illness severity. The references list of each of these articles was also scrutinized for identifying other eligible documents. Mean and standard deviation (SD) of MR-proADM values were included in a pooled analysis, with estimation of weighted mean difference (WMD) and 95\% confidence interval (95\% CI) in COVID-19 patients with or without critical illness. When mean value and SD were unavailable, they were estimated from sample size, median and interquartile range (IQR), as suggested by Hozo et al. [6]. When multiple MR-proADM values were shown, those corresponding to COVID-19 peak severity were selected. A quality effects model was used for pooled analysis, whilst a second random effects model was also calculated to adjust for heterogeneity emerging across different studies. Heterogeneity was evaluated with the $\chi^{2}$ test and $I^{2}$ statistic. The statistical analysis was performed using MetaXL, software Version 5.3 (EpiGear International Pty Ltd., Sunrise Beach, Australia). The study was conducted in agreement with the declaration of Helsinki and within the terms of local legislation.

The electronic search carried out in accordance the above-mentioned criteria identified 34 documents after elimination of duplicates. Among these, 28 were excluded as they were review articles $(n=13)$, editorial material $(n=1)$ or correspondence without original data $(n=2)$, did not specifically deal with COVID-19 $(n=6)$, did not provide MR-proADM values $(n=2)$, lack of complete information on MR-proADM values $(n=1)$, or MR-proADM values were not stratified according to COVID-19 severity $(n=3)$. No significant disagreement emerged between 
Table 1 Summary of clinical studies which explored mid-regional pro-adrenomedullin (MR-proADM) levels in coronavirus disease 2019 (COVID-19) patients with or without critical illness

\begin{tabular}{|c|c|c|c|c|}
\hline Authors & Setting & Sample size & Endpoint & $\begin{array}{l}\text { Values (severe vs. non- } \\
\text { severe; nmol/L) }\end{array}$ \\
\hline Gregoriano C et al. (2021) [7] & Switzerland & 89 (19\% severe) & Death & $1.50 \pm 0.40$ vs. $0.85 \pm 0.23$ \\
\hline Montrucchio G et al. (2021) [8] & Italy & 57 (54\% severe) & ICU admission or death & $2.37 \pm 1.63$ vs. $1.13 \pm 1.16$ \\
\hline Popov DA et al. (2020) [9] & Russia & 97 (14\% severe) & Death & $1.25 \pm 0.31$ vs. $0.78 \pm 0.22$ \\
\hline Roedl K. et al. (2021) [10] & Germany & 64 (45\% severe) & RRT & $2.46 \pm 0.64$ vs. $1.34 \pm 0.39$ \\
\hline Sozio E et al. (2021) [11] & Italy & $111(25 \%$ severe $)$ & Death or intubation & $1.36 \pm 0.31$ vs. $0.74 \pm 0.23$ \\
\hline Spoto S et al. (2021) [12] & Italy & 69 (58\% severe) & ARDS & $2.30 \pm 1.11$ vs. $1.12 \pm 0.45$ \\
\hline
\end{tabular}

$A R D S$ acute respiratory distress syndrome, $I C U$ intensive care unit, $R R T$ renal replacement therapy

the two reviewers. Six studies were thus finally included in pooled analysis, totaling 487 COVID-19 patients, 159 (32.6\%) with critical illness, as summarized in Table 1 [7-12]. All included studies were cross sectional investigations, three conducted in Italy, while the others were located in Germany, Russia and Switzerland. The clinical endpoints used for characterizing critical illness of COVID-19 were cumulative mortality in two studies, as opposed to intensive care unit (ICU) admission or death, need for renal replacement therapy (RRT), death or intubation, and acute respiratory distress syndrome (ARDS) in the remaining investigations (Table 1). The pooled analysis of these six studies is shown in Fig. 1, demonstrating a positive difference of MR-proADM values between patients with or without critical COVID-19 in each individual study. The WMD of MR-proADM values in COVID-19 patients with critical illness versus those without was $0.67(95 \%$ CI $0.42-0.93) \mathrm{nmol} / \mathrm{L}$ in the quality effects model (with high heterogeneity, $I^{2}=81 \%$ ) (Fig. 1), further increasing to $0.80(95 \% \mathrm{CI} 0.58-1.02) \mathrm{nmol} / \mathrm{L}$ in the random effects model. Overall, MR-proADM values were found to be increased by $74 \%$ (95\% CI 46-103\%) in COVID-19 patients with critical illness compared to those without.
Notably, two studies among those excluded deserve special mention. Hupf et al. assayed ADM gene expression rather than the circulating hormone concentration in 21 COVID-19 patients and, in agreement with our findings on MR-proADM levels, they reported significantly higher values in subjects who died than in those who survived [13]. In a subsequent investigation, which could not be included in the pooled analysis due to lack of complete information on MR-proADM values, Benedetti et al. found that the median concentration of this biomarker was significantly higher in COVID-19 patients who died $(11 / 21 ; 52 \%)$ that in those who survived ( $3.5 \mathrm{vs.} 0.8 \mathrm{nmol} / \mathrm{L} ; p=0.006)$ [14]. As concerns the possible mechanisms underpinning the negative effect of MR-proADM on infectious diseases course, including COVID-19, this molecule is deeply involved in the inflammatory response and in progression from sepsis to septic shock [1]. In both conditions, vascular endothelium represent the most predominant source of MR-proADM, although other organs may contribute to its production. Since endothelial injury is commonplace in patients with COVID-19, and progression to critical illness is always associated with multiple organ failure [15], MRproADM assessments may hence represent a valuable tool for monitoring disease severity and stratifying the risk of critical illness or death.
Fig. 1 Weighted mean difference (WMD) and 95\% confidence interval $(95 \% \mathrm{CI})$ of mid-regional pro-adrenomedullin (MR-proADM) in patients with coronavirus disease 2019 (COVID-19) with or without critical illness

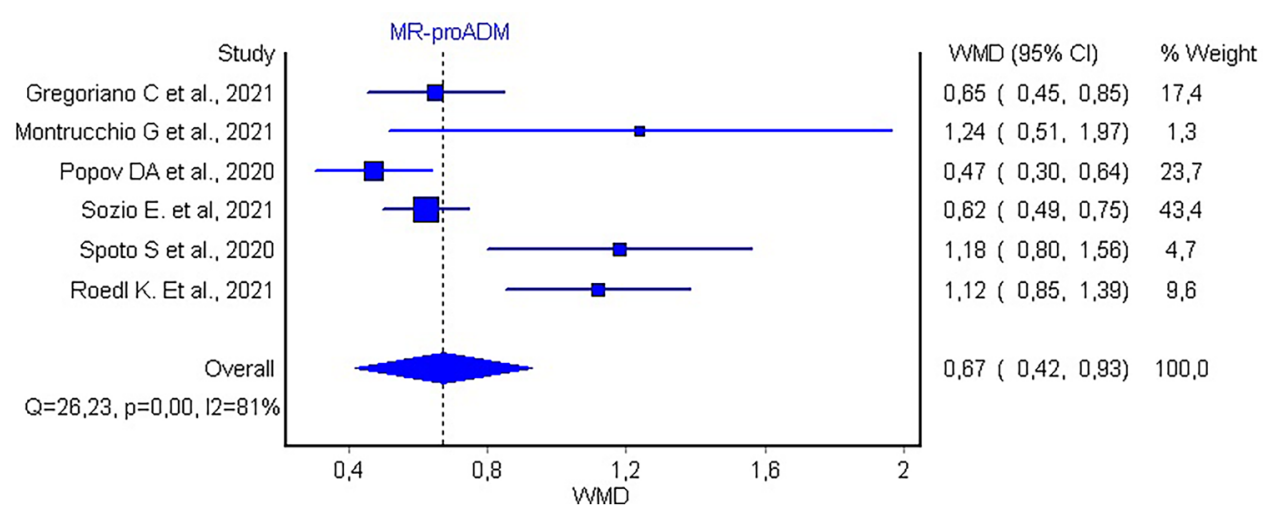


Funding No financial support was provided relevant to this article.

\section{Declarations}

Conflict of interest All authors report no conflicts of interest relevant to this article.

Ethical approval Not applicable.

Human and animal rights Not applicable.

Informed consent Not applicable.

\section{References}

1. Önal U, Valenzuela-Sánchez F, Vandana KE et al (2018) Midregional pro-adrenomedullin (MR-proADM) as a biomarker for sepsis and septic shock: narrative review. Healthcare (Basel) 6:110

2. Valenzuela-Sánchez F, Valenzuela-Méndez B, Rodríguez-Gutiérrez JF et al (2016) New role of biomarkers: mid-regional proadrenomedullin, the biomarker of organ failure. Ann Transl Med 4:329

3. Liu D, Xie L, Zhao H et al (2016) Prognostic value of midregional pro-adrenomedullin (MR-proADM) in patients with community-acquired pneumonia: a systematic review and metaanalysis. BMC Infect Dis 16:232

4. Bello S, Lasierra AB, Mincholé E et al (2012) Prognostic power of proadrenomedullin in community-acquired pneumonia is independent of aetiology. Eur Respir J 39:1144-1155

5. Zhang H, Zhang Y, Wu J et al (2020) Risks and features of secondary infections in severe and critical ill COVID-19 patients. Emerg Microbes Infect 9:1958-1964

6. Hozo SP, Djulbegovic B, Hozo I (2005) Estimating the mean and variance from the median, range, and the size of a sample. BMC Med Res Methodol 5:13
7. Gregoriano C, Koch D, Kutz A et al (2021) The vasoactive peptide MR-pro-adrenomedullin in COVID-19 patients: an observational study. Clin Chem Lab Med 59:995-1004

8. Montrucchio G, Sales G, Rumbolo F et al (2021) Effectiveness of mid-regional pro-adrenomedullin (MR-proADM) as prognostic marker in COVID-19 critically ill patients: an observational prospective study. PLoS ONE 16:e02

9. Popov DA, Borovkova UL, Rybka MM et al (2020) Predictive value of proadrenomedullin in patients with COVID-19. Anesteziol Reanimatol 6:6-12

10. Roedl K, Jarczak D, Fischer M et al (2021) MR-proAdrenomedullin as predictor of renal replacement therapy in a cohort of critically ill patients with COVID-19. Biomarkers. https://doi.org/10. 1080/1354750X.2021.1905067 (Epub ahead of print)

11. Sozio E, Tascini C, Fabris M, D’Aurizio F, De Carlo C, Graziano E, Bassi F, Sbrana F, Ripoli A, Pagotto A, Giacinta A, Gerussi V, Visentini D, De Stefanis P, Merelli M, Saeed K, Curcio F (2021) MR-proADM as prognostic factor of outcome in COVID-19 patients. Sci Rep 11:5121

12. Spoto S, Agrò FE, Sambuco F et al (2021) High value of midregional proAdrenomedullin in COVID-19: a marker of widespread endothelial damage, disease severity and mortality. J Med Virol 93:2820-2827

13. Hupf J, Mustroph J, Hanses F et al (2020) RNA-expression of adrenomedullin is increased in patients with severe COVID-19. Crit Care 24:527

14. Benedetti I, Spinelli D, Callegari T, Bonometti R, Molinaro E, Novara E, Cassinari M, Frino C, Guaschino R, Boverio R, Lauritano EC (2021) High levels of mid-regional proadrenomedullin in ARDS COVID-19 patients: the experience of a single, Italian center. Eur Rev Med Pharmacol Sci 25:1743-1751

15. Lippi G, Sanchis-Gomar F, Henry BM (2020) COVID-19: unravelling the clinical progression of nature's virtually perfect biological weapon. Ann Transl Med 8:693

Publisher's Note Springer Nature remains neutral with regard to jurisdictional claims in published maps and institutional affiliations. 